\title{
Frame-indifferent and positive-definite Reynolds stress-strain relation
}

\author{
By LIQIU WANG \\ Department of Mechanical Engineering, University of Hong Kong, \\ Pokfulam Road, Hong Kong \\ (Received 2 December 1996 and in revised form 25 August 1997)
}

Both necessary and sufficient conditions are derived in a systematic, rigorous way for a Reynolds stress model to preserve both frame indifference and positive semidefiniteness of the Reynolds stresses and to satisfy the principle of material frame indifference (PMFI). Also developed are two approximate theories, a linear theory and a quadratic theory, to simplify the work of developing models satisfying such conditions. The results are also valid for the higher-order correlations and the SGS Reynolds stresses. This leads to the results either confirming the previous intuitive arguments or offering new insights into turbulence modelling, and is of significance in clarifying some controversies in the literature, examining how well existing models preserve the physics, and developing new models.

An examination of various existing models with respect to the necessary and sufficient conditions obtained in the present work shows that no one model can simultaneously satisfy the invariance, the realizability and the PMFI. This offers answers to some fundamental questions regarding the existing models, such as the reason why $k-\varepsilon$ and $k-l$ models fail to give accurate predictions for the normal Reynolds stresses.

\section{Introduction}

Reynolds stresses are believed to be quantities determined by the mean velocity field. Based on this fundamental intrinsic belief, various approaches have been proposed to relate the Reynolds stresses to the mean velocity field, so-called turbulence modelling. The readers are referred to Lumley (1990), Speziale (1991) and Gatski, Sarkar \& Speziale (1992) for some excellent reviews and discussions of this important topic. While some results of turbulence calculations obtained based on some commonly used models seem encouraging, they fail to meet either one or both of two natural fundamental requirements for turbulence models: preserving the fundamental properties of the quantities being modelled and satisfying some classical principles regarding the behaviour of materials.

The modelling of the Reynolds stresses consists of replacing them by constitutive equations expressing them as functions of the mean velocity field. While such constitutive equations may take different forms such as algebraic and differential, it appears to be a basic requirement to preserve the properties which the Reynolds stresses hold by their definition. Two such properties are the frame indifference (Speziale 1979) and the positive semi-definiteness found by the pioneering work of Schumann (1977). The former follows from the definition of the Reynolds stress and states that the Reynolds stress is a second-order tensor $\boldsymbol{R}$ which transforms frame-indifferent spatial vectors 
into frame-indifferent spatial vectors. The latter comes from the fact that the velocity field is a real-valued field and $R_{i j}$ is a linear function of velocity products $v_{i} v_{j}$ and indicates that $\boldsymbol{R}$ satisfies $\boldsymbol{a} \cdot \boldsymbol{R} \boldsymbol{a} \geqslant 0$ for all vectors $\boldsymbol{a}$ in the Euclidean vector space. (Vreman, Geurts \& Kuerten (1994) have discussed a number of reasons for the preference of a positive semi-definite subgrid-scale (SGS) Reynolds stress tensor in large-eddy simulations, and have found that positiveness of the filter function forms a necessary and sufficient requirement.) We can also show that the positive semidefiniteness of $\boldsymbol{R}$ follows logically from the second law of thermodynamics (Wang 1997). The issues concerned with whether a model guarantees these two properties are referred as the invariance and the realizability in the literature, respectively. In Schumann (1977), Speziale $(1979,1980,1984,1985,1987)$ and Speziale, Abid \& Durbin (1993), some commonly used models were examined and it was found that they were incapable of preserving the frame indifference and/or the positive semi-definiteness. This led to the development of improved models mainly in the context of second-order closure modelling (Schumann 1977; Speziale 1987; Speziale, Sarkar \& Gatski 1991; Gatski \& Speziale 1993; Durbin \& Speziale 1994; Speziale, Abid \& Durbin 1994).

While the first requirement focuses on the properties of the Reynolds stresses themselves, the second requirement emphasizes the relation of $\boldsymbol{R}$ with the mean velocity fields. The purpose of Reynolds stress modelling is to develop its constitutive equations (Lumley 1970). Such equations are supposed to take account of the behaviour of materials, and to be independent of conservation laws of mass, linear momentum, rotational momentum and energy, etc. This is quite similar to those for the Cauchy stresses in continuum mechanics. The constitutive relations for materials in modern continuum mechanics are required to satisfy three principles which come from our everyday experience in observing materials in deformation and motion (Truesdell 1966): the principles of determinism, local action and material frame indifference. The first states that the stress of a body is determined by the history of the motion which the body has undergone. The second indicates that the motion of the material outside an arbitrarily small neighbourhood of a material particle may be ignored in determining the stress acting at this particle. The third principle states that all physical laws, definitions and descriptions of material behaviour which hold in a dynamic process are the same for every observer, i.e. in every frame of reference.

The validity of the first principle for the Reynolds stresses is well accepted. While some experimental results provide evidence that the Reynolds stress is determined in a neighbourhood with radius of the order of an integral scale (Lumley 1970), especially in strongly inhomogeous flows (Speziale 1991), the principle of local action is still accepted to be valid at least in an approximate sense (in particular for a nearly homogeneous turbulence flow), and is used in nearly all current models (Pope 1975; Lumley 1990; Speziale 1991; Xu \& Speziale 1996).

There is a controversy in the literature with regard to the applicability of the principle of material frame indifference (we refer this principle as PMFI hereinafter) to the turbulence models (Lumley 1983; Speziale 1984; Lumley 1990; Ristocelli, Lumley \& Abid 1995). This is believed to be due to the overlooking of the applicability condition of the PMFI. In the literature (e.g. Speziale 1980; Lumley 1970, 1983), the frame dependency of the Navier-Stokes equations and/or the Reynolds stress transport equations is, usually, used to judge the applicability of the PMFI to the turbulence models (in particular to conclude that the PMFI is invalid). This is clearly improper since the PMFI holds only for the physical laws and relations regarding the material behaviour. It is not necessarily true for the other laws such as Newton's second law of motion, which describes the motion of a body rather than the material 
behaviour. This law is valid only for the inertial frame of reference (Morris 1981). This can be understood by noting that it proportionally relates the force, a frame-indifferent vector, to the acceleration, a frame-dependent vector through mass, a frame-indifferent scalar. The Navier-Stokes equations and the Reynolds stress transport equations $\dagger$ come from Newton's second law of motion, are valid only for the inertial frame of reference (Morris 1981), and are unable to provide information about the Reynolds stresses in a non-inertial frame of reference. In addition to the justification in the literature (Speziale 1979, 1980, 1981, 1983, 1984; Pope 1983; Ristocelli et al. 1995, etc.), we accept the PMFI also for the following reasons: (i) we expect to develop models with sufficient generality: any model violating the PMFI cannot be a general model; (ii) somewhere in our theoretical structure we must incorporate information about the behaviour of materials which satisfies the PMFI, if not for the Reynolds stress then we must impose it on the higher-order turbulence correlations.

An examination of the existing models revealed that some of them violate the PMFI while nearly all satisfy the principles of determinism and local action (Speziale 1979, 1980, 1987, 1989, 1991; Speziale et al. 1991; Gatski \& Speziale 1993). This has motivated the development of improved models mainly in the context of the second-order closure models (Speziale 1979, 1980, 1987; Speziale et al. 1991; Gatski \& Speziale 1993; Ristorcelli et al. 1995).

Typical previous works dedicated to developing models preserving the frame indifference and/or the positive semi-definiteness and/or satisfying the PMFI are Schumann (1977), Speziale (1979, 1980, 1987), Speziale et al. (1991), Gatski \& Speziale (1993), Durbin \& Speziale (1994), Speziale et al. (1994), and Ristorcelli et al. (1995). While these works have uncovered some important features of invariance, realizability or PMFI, questions concerning both necessary and sufficient conditions for a model to preserve both frame indifference and positive semi-definiteness and satisfy the PMFI have been left unanswered. Also, because of the complexities of the problem, the arguments used in the previous works are mainly intuitive in nature, focusing on modifying the existing models by obtaining some sufficient conditions for the invariance, realizability or PMFI (Speziale 1987). It appears necessary to re-examine some concepts and methods in the literature. For example the frame indifference of the Reynolds stresses and the higher-order correlations, the PMFI, and the principle of observer transformation (Truesdell 1977) are sometimes confused in the literature (e.g. Speziale 1979, 1980). The analysis of the invariance of Navier-Stokes equations and the Reynolds stress transport equations (e.g. Speziale 1979, 1989) is also invalid since such equations are only valid for the inertial frame of reference. Consequently, the results regarding the invariance of the models based on such an analysis and new models developed to make the Reynolds stress transport equations frame indifferent should be re-examined.

The methods of implementing the frame indifference and the PMFI in the literature also seem questionable. In particular, the choice of $\boldsymbol{D}$ (the velocity strain tensor of the mean velocity) and $\boldsymbol{W}$ (the vorticity tensor of the mean velocity) as two independent variables when applying Smith's representation theorem of isotropic functions (Smith 1971) appears to lead to some controversial results such as the dependence of the Reynolds stresses and higher turbulence correlations on the vorticity tensor $\boldsymbol{W}$ (Pope 1975; Speziale et al. 1991; Gatski \& Speziale 1993). (Readers are referred to Speziale (1980), Speziale et al. (1991), Gatski \& Speziale (1993) and Xu \& Speziale (1996) for

$\dagger$ These equations relate cause (Reynolds stress) to effect (fluid motion), and are dynamic rather than constitutive relations. We cannot expect a validation of the PMFI for them. 
details of this controversy.) An examination of Smith's representation theorem shows its applicability conditions: (i) the function must be a symmetric or skew tensorvalued isotropic function; (ii) all independent variables must be symmetric tensors, skew tensors or vectors; and (iii) the independent variables must be independent of each other. Note that $\boldsymbol{D}$ and $\boldsymbol{W}$ cannot be considered to be independent. The turbulent kinetic energy $k$, the length scale of turbulence $l$ (or the turbulence dissipation rate $\varepsilon$ ), and mean velocity gradients cannot be considered as the independent variables either (Speziale 1987).

It is well accepted in the turbulence literature that the Reynolds stresses are related to the rates of strain and local scalar quantities (Pope 1975). However, a rigorous analysis is not available regarding what should be used as such characteristic scalars and how many scalar quantities are required. Based on some intuitive assumptions and dimensional arguments (Lumley 1970; Pope 1975), $k$ and $l$ (or $\varepsilon$ ) are usually used as two characteristic scalars in the literature. This lacks justification, and leads the models developed to be implicit since $k$ is function of the Reynolds stresses. While $k$, $l(\varepsilon)$ are of physical significance, they may not necessarily be characteristic scalars in relating the Reynolds stresses to the mean flow fields.

The motivation for the present work comes from the desire to derive both necessary and sufficient conditions in a systematic rigorous way for a Reynolds stress model to preserve both frame indifference and the positive semi-definiteness of the Reynolds stresses and to satisfy the PMFI. In the derivation, no intuitive assumptions are introduced. This leads to some conclusive results. Among them, some confirm the previous intuitive arguments, and others form new insights into turbulence modelling. Also developed in the present work are two approximate theories to simplify the development of specific models satisfying both necessary and sufficient conditions for the invariance, realizability and PMFI. Although some results may be known to some readers, the approach to obtaining them is believed to be more systematic and rigorous. A similar analysis can been carried out for the high-order turbulence correlations and the SGS Reynolds stresses occurring in large-eddy simulation (LES) (Mason 1994; Ciofalo 1994; Lesieur \& Métais 1996).

As a general approach is developed for establishing physics-preserving turbulence closure models, it is necessary to construct the theory from first principles incorporating as much physics as possible. A complete physics-preserving model of turbulence has thus to include (i) invariance, (ii) realizability, (iii) PMFI, (iv) effect of memory, (v) non-locality, and even (vi) non-determinism of the mean flow properties, in particular, for large-eddy simulation cases (Mason 1994; Schumann 1995). This complicates the analysis so much that new mathematical tools have to be developed first. Within the scope of the present work, i.e. deriving both necessary and sufficient conditions for a model to satisfy the first three requirements, we impose both the principle of determinism (without taking account of the effect of memory) and the principle of local action on our constitutive relations. The results are therefore limited to nearly homogenous flows with negligible effect of memory. The readers are referred to Mason (1994) and Schumann (1995) for a detailed discussion on the physical aspects of non-locality etc. No attempt is made in the present work to develop specific models through experimentally determining the coefficients in the general model.

Though the present work develops, from essentially first principles, a more general model which forms both necessary and sufficient conditions for the invariance, realizability and PMFI, the author does not see it as detracting in any way from the value of the previous works on this subject. Those works continue to be of interest and importance as they pioneered such an important and difficult subject, and the 
previous models can be used for certain turbulence flows by noting that all specific models were developed by determining the coefficients through experiments.

\section{Frame indifference of Reynolds stresses and PMFI}

Consider a class of constitutive relations which relate $\boldsymbol{R}$ to $\boldsymbol{v}, \boldsymbol{L}$ and the other scalar-valued parameters which are independent of $\boldsymbol{v}$ and $\boldsymbol{L}$, i.e.

$$
\boldsymbol{R}=\boldsymbol{F}(\mathrm{OP}, \boldsymbol{v}, \boldsymbol{L}) .
$$

Here $\boldsymbol{R}$ is a second-order tensor which can be Reynolds stresses, SGS Reynolds stresses or the higher turbulence correlations; $\boldsymbol{v}$ is a vector-valued variable in the Euclidean vector space and can be the mean velocity vector (ensemble or time mean), or filtered velocity vector; $\boldsymbol{L}$ is a second-order tensor-valued variable which is the velocity gradient tensor of $\boldsymbol{v}$; OP denotes the other scalar-valued thermophysical parameters which are independent of $\boldsymbol{v}$ and $\boldsymbol{L}$ and are typically the local thermodynamic state variables; $\boldsymbol{F}$ is a second-order tensor-valued function.

In sharp contrast to the literature (Pope 1975; Speziale et al. 1991; Gatski \& Speziale 1993), we choose $L$ as an independent variable instead of its symmetric part $\boldsymbol{D}$ (the velocity strain tensor) and skew part $\boldsymbol{W}$ (the vorticity tensor) because $\boldsymbol{D}$ and $\boldsymbol{W}$ cannot be regarded as independent. We do not include $k, l$ or $\varepsilon$ as the independent variables, and do not restrict $\boldsymbol{F}$ to be symmetric or skew tensor-valued either. The exclusion of the explicit dependence of $\boldsymbol{R}$ on time $t$ and position vector $\boldsymbol{r}$ comes from the fact that they affect $\boldsymbol{R}$ through OP, $\boldsymbol{v}$ and $\boldsymbol{L}$.

The constitutive relation (1) satisfies both the principle of determinism and the principle of local action since we assume that $\boldsymbol{R}$ at a point is a function of OP, $\boldsymbol{v}$ and $\boldsymbol{L}$ at that point. It is also quite general in the sense that the effects of memory and higher orders of $\boldsymbol{L}$ such as $\boldsymbol{L}$ and $\nabla \boldsymbol{L}$ are usually not significant. An analysis for a more general constitutive relation permitting the dependence on such higher orders of $\boldsymbol{L}$ and taking account of the effects of memory and non-locality of turbulence will be the topic of future research.

\subsection{Necessary conditions}

\subsection{1. $\boldsymbol{R}-\boldsymbol{v}$ relation}

THEOREM 1. $\boldsymbol{R}$ is independent of $\boldsymbol{v}$.

Proof. For a second observer *, the PMFI and the frame indifference of $\boldsymbol{R}$ together require that

$$
\begin{gathered}
\boldsymbol{R}^{*}=\boldsymbol{F}\left((\mathrm{OP})^{*}, \boldsymbol{v}^{*}, \boldsymbol{L}^{*}\right), \\
\boldsymbol{R}^{*}=\boldsymbol{Q}(t) \boldsymbol{R} \boldsymbol{Q}^{T}(t),
\end{gathered}
$$

in which superscript $*$ represents the quantities observed by observer $*$; and $\mathbf{Q}$ is an arbitrary rotation tensor.

From the principle of observer transformations (Truesdell 1977),

$$
\left.\begin{array}{l}
(\mathrm{OP})^{*}=\mathrm{OP}, \\
\boldsymbol{r}^{*}=\mathbf{Q}(t) \boldsymbol{r}+\boldsymbol{c}(t), \\
\boldsymbol{v}^{*}=\mathrm{d} \boldsymbol{r}^{*} / \mathrm{d} t=\mathbf{Q}(t) \boldsymbol{r}+\mathbf{Q}(t) \boldsymbol{v}+\dot{\boldsymbol{c}}(t), \\
\mathbf{L}^{*}=\boldsymbol{Q}(t) \boldsymbol{L} \boldsymbol{Q}^{T}(t)+\dot{\boldsymbol{Q}}(t) \mathbf{Q}^{T}(t),
\end{array}\right\}
$$


where $\boldsymbol{r}$ is a position vector of a material point; $\boldsymbol{c}(t)$ is an arbitrary vector-valued function of time $t$; and a dot over a letter indicates a time derivative.

On substitution of (1), (3) and (4) into (2), we have that (suppressing $t$ )

$$
\mathbf{Q} \boldsymbol{F}(\mathrm{OP}, \boldsymbol{v}, \boldsymbol{L}) \mathbf{Q}^{T}=\boldsymbol{F}\left(\mathrm{OP}, \dot{\mathbf{Q}} \boldsymbol{r}+\boldsymbol{Q} \boldsymbol{v}+\dot{\boldsymbol{c}}, \mathbf{Q} \mathbf{L} \mathbf{Q}^{T}+\dot{\mathbf{Q}} \mathbf{Q}^{T}\right) \quad \forall \mathbf{Q} \text { and } \boldsymbol{c}
$$

which indicates that $\boldsymbol{F}$ is not an isotropic function of $\boldsymbol{v}$ and $\boldsymbol{L}$ in general.

Since (5) holds for all $\boldsymbol{Q}$, it must be true for $\boldsymbol{Q}=\mathbf{1}$. Take $\boldsymbol{Q}=\mathbf{1}$, then $\dot{\boldsymbol{Q}}=\mathbf{0}$. Equation (5) thus yields

$$
\boldsymbol{F}(\mathrm{OP}, \boldsymbol{v}, \boldsymbol{L})=\boldsymbol{F}(\mathrm{OP}, \boldsymbol{v}+\dot{\boldsymbol{c}}, \boldsymbol{L}) \quad \forall \dot{\boldsymbol{c}} .
$$

This implies that $\boldsymbol{F}$ is independent of $\boldsymbol{v}$.

By applying Theorem 1, (1) and (5) reduce to

$$
\begin{gathered}
\boldsymbol{R}=\boldsymbol{F}(\mathrm{OP}, \boldsymbol{L}), \\
\mathbf{Q} \boldsymbol{F}(\mathrm{OP}, \boldsymbol{L}) \boldsymbol{Q}^{T}=\boldsymbol{F}\left(\mathrm{OP}, \boldsymbol{Q} \mathbf{L} \mathbf{Q}^{T}+\dot{\mathbf{Q}} \mathbf{Q}^{T}\right) \quad \forall \boldsymbol{Q} .
\end{gathered}
$$

\subsection{2. $\boldsymbol{R}-\boldsymbol{L}$ relation}

Note that $\boldsymbol{L}$ can be uniquely decomposed into a symmetric tensor $\boldsymbol{D}$ (the velocity strain tensor) and a skew tensor $\boldsymbol{W}$ (the vorticity tensor). Expression (8) may then be rewritten as

$$
\boldsymbol{Q} \boldsymbol{F}(\mathrm{OP}, \mathbf{L}) \boldsymbol{Q}^{T}=\boldsymbol{F}\left(\mathrm{OP}, \mathbf{Q} \boldsymbol{D} \boldsymbol{Q}^{T}+\mathbf{Q} \mathbf{W} \boldsymbol{Q}^{T}+\dot{\boldsymbol{Q}} \boldsymbol{Q}^{T}\right) \quad \forall \boldsymbol{Q} .
$$

Theorem 2. For any fixed time $\tau$ and all time $t$,

$$
\boldsymbol{Q}(t)=\exp [\hat{\boldsymbol{\Omega}}(t-\tau)]=\sum_{n=0}^{\infty} \frac{(t-\tau)^{n}}{n !} \hat{\boldsymbol{\Omega}}^{n}
$$

is a rotation tensor provided that $\hat{\boldsymbol{\Omega}}$ is a fixed (time-independent) skew tensor.

Proof. From the theory of series, it is well known that the series

$$
\sum_{n=0}^{\infty} \frac{(t-\tau)^{n}}{n !} \hat{\boldsymbol{\Omega}}^{n}
$$

is absolutely convergent to $\exp [\hat{\boldsymbol{\Omega}}(t-\tau)]$.

Let

$$
\boldsymbol{Q}(t)=\exp [\hat{\boldsymbol{\Omega}}(t-\tau)]=\sum_{n=0}^{\infty} \frac{(t-\tau)^{n}}{n !} \hat{\boldsymbol{\Omega}}^{n}
$$

Then

$$
\begin{gathered}
\boldsymbol{Q}(\tau)=\mathbf{1}, \\
\dot{\boldsymbol{Q}}(t)=\frac{\mathrm{d} \boldsymbol{Q}(t)}{\mathrm{d} t}=\hat{\boldsymbol{\Omega}}+(t-\tau) \hat{\boldsymbol{\Omega}}^{2}+\frac{1}{2}(t-\tau)^{2} \hat{\boldsymbol{\Omega}}^{3}+\cdots \\
=\hat{\boldsymbol{\Omega}}\left[\mathbf{1}+(t-\tau) \hat{\boldsymbol{\Omega}}+\frac{1}{2}(t-\tau)^{2} \hat{\boldsymbol{\Omega}}^{2}+\frac{1}{6}(t-\tau)^{3} \hat{\boldsymbol{\Omega}}^{3}+\cdots\right]=\hat{\boldsymbol{\Omega}} \mathbf{Q}(t)
\end{gathered}
$$

and

$$
\left(\boldsymbol{Q}^{T} \boldsymbol{Q}\right)^{\cdot}=\boldsymbol{Q}^{T}\left(\hat{\boldsymbol{\Omega}}+\hat{\boldsymbol{\Omega}}^{T}\right) \boldsymbol{Q}=\mathbf{0}
$$


if $\hat{\boldsymbol{\Omega}}$ is a skew tensor. Therefore

$$
\boldsymbol{Q}^{T}(t) \boldsymbol{Q}(t)=\boldsymbol{Q}^{T}(\tau) \boldsymbol{Q}(\tau)=\mathbf{1} \quad \forall t .
$$

Since $(\operatorname{det} \mathbf{G})=\operatorname{det} \boldsymbol{G} \operatorname{tr}\left(\dot{\boldsymbol{G}} \mathbf{G}^{-1}\right)$ for all invertible $\boldsymbol{G}$, then

$$
(\operatorname{det} \boldsymbol{Q})^{\circ}=\operatorname{det} \boldsymbol{Q} \operatorname{tr}\left(\dot{\boldsymbol{Q}} \boldsymbol{Q}^{T}\right)=\operatorname{det} \boldsymbol{Q} \operatorname{tr}\left(\hat{\boldsymbol{\Omega}} \boldsymbol{Q} \boldsymbol{Q}^{T}\right)=\operatorname{det} \boldsymbol{Q} \operatorname{tr}(\hat{\boldsymbol{\Omega}})=0 .
$$

Therefore,

$$
\operatorname{det} \boldsymbol{Q}(t)=\operatorname{det} \boldsymbol{Q}(\tau)=\operatorname{det} \mathbf{1}=1 \quad \forall t .
$$

Expressions (10) and (11) are the mathematical form of Theorem 2.

THEOREM 3. For rotation tensor $\boldsymbol{Q}(t)=\exp [\hat{\boldsymbol{\Omega}}(t-\tau)] \hat{\mathbf{Q}}$, at any instant $\tau$, we can take $\mathbf{Q}(\tau)$ and $\mathbf{Q}(\tau) \mathbf{Q}^{T}(\tau)$ to be arbitrary independent rotation and skew tensors respectively, where $\hat{\boldsymbol{Q}}$ is any fixed rotation tensor, and $\hat{\boldsymbol{\Omega}}$ is any fixed skew tensor.

Proof. Since both $\hat{\boldsymbol{Q}}$ and $\exp [\hat{\boldsymbol{\Omega}}(t-\tau)]$ are rotation tensors (Theorem 2), then $\boldsymbol{Q}(t)=\exp [\hat{\boldsymbol{\Omega}}(t-\tau)] \hat{\mathbf{Q}}$ is also a rotation tensor for all time $t$. Also,

$$
\begin{gathered}
\mathbf{Q}(\tau)=\hat{\mathbf{Q}}, \\
\dot{\mathbf{Q}}(\tau) \boldsymbol{Q}^{T}(\tau)=\hat{\boldsymbol{\Omega}} \boldsymbol{Q}(\tau) \boldsymbol{Q}^{T}(\tau)=\hat{\boldsymbol{\Omega}} .
\end{gathered}
$$

They are clearly independent rotation and skew tensors if $\hat{\boldsymbol{Q}}$ and $\hat{\boldsymbol{\Omega}}$ are arbitrary fixed rotation and skew tensors respectively.

TheOREm 4. $\boldsymbol{L}$ affects $\boldsymbol{R}$ only through the velocity strain tensor $\boldsymbol{D}$.

Proof. To prove this, choose $\boldsymbol{Q}(t)$ defined in Theorem 3 as the rotation tensor in (9), while for any instant $\tau,-\left.\mathbf{Q} \boldsymbol{W} \boldsymbol{Q}^{T}\right|_{\tau}$ is used as the skew tensor $\hat{\boldsymbol{\Omega}}$, i.e. $\hat{\boldsymbol{\Omega}}=-\left.\mathbf{Q} \boldsymbol{W} \boldsymbol{Q}^{T}\right|_{\tau}$ (such a $\hat{\boldsymbol{\Omega}}$ is a skew tensor since $\hat{\boldsymbol{\Omega}}^{T}=-\left.\boldsymbol{Q} \boldsymbol{W}^{T} \boldsymbol{Q}^{T}\right|_{\tau}=\left.\boldsymbol{Q} \boldsymbol{W} \boldsymbol{Q}^{T}\right|_{\tau}=-\hat{\boldsymbol{\Omega}}$ ). Then at time $t=\tau,(9)$ yields

$$
\hat{\mathbf{Q}} \boldsymbol{F}(\mathrm{OP}, \boldsymbol{L}) \hat{\mathbf{Q}}^{T}=\boldsymbol{F}\left(\mathrm{OP}, \hat{\mathbf{Q}} \boldsymbol{D} \hat{\mathbf{Q}}^{T}\right) \quad \forall \hat{\mathbf{Q}} .
$$

Since this is true for all fixed rotation tensors $\hat{\mathbf{Q}}$, it must hold for $\hat{\mathbf{Q}}=\mathbf{1}$. Let $\hat{\mathbf{Q}}=\mathbf{1}$, then (14) leads to

$$
\boldsymbol{F}(\mathrm{OP}, \boldsymbol{L})=\boldsymbol{F}(\mathrm{OP}, \boldsymbol{D})
$$

or

$$
\boldsymbol{R}=\boldsymbol{F}(\mathrm{OP}, \boldsymbol{D})
$$

\subsection{3. $\boldsymbol{R}-\boldsymbol{D}$ relation}

By applying (16), the PMFI yields

$$
\boldsymbol{R}^{*}=\boldsymbol{F}\left((\mathrm{OP})^{*}, \boldsymbol{D}^{*}\right) \text {. }
$$

By applying the frame indifference of $\boldsymbol{R}$ and $\boldsymbol{D}^{*}=\boldsymbol{Q} \boldsymbol{D} \boldsymbol{Q}^{T}$ (Truesdell 1977) in (17), we obtain

$$
\boldsymbol{Q} \boldsymbol{F}(\mathrm{OP}, \boldsymbol{D}) \mathbf{Q}^{T}=\boldsymbol{F}\left(\mathrm{OP}, \mathbf{Q D} \boldsymbol{Q}^{T}\right) \quad \forall \mathbf{Q}
$$

which indicates that $\boldsymbol{F}$ is an isotropic function of $\boldsymbol{D}$. However, we cannot appeal to Smith's representation theorem (Smith 1971) since we do not restrict $\boldsymbol{R}$ to be the symmetric or skew tensor. 
Since the velocity strain tensor $\boldsymbol{D}$ is a real symmetric tensor, it has three real eigenvalues. The three eigenvalues can be distinct, or identical, or two of them can be identical. In the present work, we focus on the case that the three eigenvalues are distinct. Similar results may be obtained for the other two cases. $(18)$

THEOREM 5. Each of three eigenvectors of $\boldsymbol{D}$ is also an eigenvector of $\boldsymbol{F}$ if it satisfies

Proof. Let $\left\{\boldsymbol{e}_{i}\right\}(i=1,2,3)$ be the eigenvectors of $\boldsymbol{D}$. The corresponding eigenvalues are denoted by $\lambda_{i}(i=1,2,3)$. Then, $\boldsymbol{D}$ may be represented, in its spectral form, as

$$
\boldsymbol{D}=\sum_{i=1}^{3} \lambda_{i} \boldsymbol{e}_{i} \otimes \boldsymbol{e}_{i} .
$$

As (18) holds for all rotation tensors we can take a particular rotation

$$
\mathbf{Q}=2 \boldsymbol{e}_{1} \otimes \boldsymbol{e}_{1}-\mathbf{1}
$$

to obtain a necessary condition. Such a $\mathbf{Q}$ is a rotation because

$$
\begin{gathered}
\mathbf{Q} \boldsymbol{Q}^{T}=\left(2 \boldsymbol{e}_{1} \otimes \boldsymbol{e}_{1}-\mathbf{1}\right)\left(2 \boldsymbol{e}_{1} \otimes \boldsymbol{e}_{1}-\mathbf{1}\right)=\mathbf{1}, \\
\operatorname{det} \boldsymbol{Q}=\operatorname{det}\left(2 \boldsymbol{e}_{1} \otimes \boldsymbol{e}_{1}-\mathbf{1}\right)=(-1)^{3} \operatorname{det}\left(\mathbf{1}-2 \boldsymbol{e}_{1} \otimes \boldsymbol{e}_{1}\right)=-\left(1-2 \boldsymbol{e}_{1} \cdot \boldsymbol{e}_{1}\right)=1 .
\end{gathered}
$$

For the rotation $\boldsymbol{Q}$ defined by (20), it is a simple matter to show that

$$
\begin{aligned}
\boldsymbol{Q} \boldsymbol{e}_{1} & =\boldsymbol{e}_{1}, \\
\boldsymbol{Q} \boldsymbol{e}_{2} & =-\boldsymbol{e}_{2}, \\
\boldsymbol{Q} \boldsymbol{e}_{3} & =-\boldsymbol{e}_{3},
\end{aligned}
$$

which imply that $\left\{\boldsymbol{e}_{i}\right\}(i=1,2,3)$ are the eigenvectors of $\boldsymbol{Q}$. The corresponding eigenvalues are $1,-1$, and -1 . Also, $\boldsymbol{e}_{1}$ is the unique axis of $\mathbf{Q}$. For the rotation $\boldsymbol{Q}$ defined by (20), expression (18) yields

$$
\begin{aligned}
\boldsymbol{Q} \boldsymbol{F}(\mathrm{OP}, \boldsymbol{D}) \boldsymbol{Q}^{T}=\boldsymbol{F}\left(\mathrm{OP}, \mathbf{Q D} \boldsymbol{Q}^{T}\right) & =\boldsymbol{F}\left(\mathrm{OP}, \sum_{i=1}^{3} \lambda_{i} \boldsymbol{Q} \boldsymbol{e}_{i} \otimes \boldsymbol{Q} \boldsymbol{e}_{i}\right)=\boldsymbol{F}\left(\mathrm{OP}, \sum_{i=1}^{3} \lambda_{i} \boldsymbol{e}_{i} \otimes \boldsymbol{e}_{i}\right) \\
& =\boldsymbol{F}(\mathrm{OP}, \boldsymbol{D})
\end{aligned}
$$

in which (19), (21)-(23) have been used. This yields (post-multiply by $\boldsymbol{Q}$ )

$$
\boldsymbol{Q F}(\mathrm{OP}, \boldsymbol{D})=\boldsymbol{F}(\mathrm{OP}, \boldsymbol{D}) \boldsymbol{Q} .
$$

Thus

$$
\boldsymbol{Q F}(\mathrm{OP}, \boldsymbol{D}) \boldsymbol{e}_{1}=\boldsymbol{F}(\mathrm{OP}, \boldsymbol{D}) \boldsymbol{Q} \boldsymbol{e}_{1}=\boldsymbol{F}(\mathrm{OP}, \boldsymbol{D}) \boldsymbol{e}_{1}
$$

which implies that

$$
\boldsymbol{F}(\mathrm{OP}, \boldsymbol{D}) \boldsymbol{e}_{1}=\mu_{1} \boldsymbol{e}_{1}
$$

for some scalar $\mu_{1}$ since $\boldsymbol{e}_{1}$ is the unique axis of $\boldsymbol{Q}$. This shows that $\boldsymbol{e}_{1}$ is an eigenvector of $\boldsymbol{F}(\mathrm{OP}, \boldsymbol{D})$.

Similarly, we can show that $\boldsymbol{e}_{2}$ and $\boldsymbol{e}_{3}$ are the other two eigenvectors of $\boldsymbol{F}$ by taking $\boldsymbol{Q}=2 \boldsymbol{e}_{2} \otimes \boldsymbol{e}_{2}-\mathbf{1}$ and $\boldsymbol{Q}=2 \boldsymbol{e}_{3} \otimes \boldsymbol{e}_{3}-\mathbf{1}$ respectively. This leads to the conclusion that $\left\{\boldsymbol{e}_{i}\right\}(i=1,2,3)$ are the eigenvectors of $\boldsymbol{F}$. 
Applying Theorem 5, $\boldsymbol{F}$ can be represented as

$$
\boldsymbol{F}(\mathrm{OP}, \boldsymbol{D})=\sum_{i=1}^{3} \mu_{i}(\mathrm{OP}, \boldsymbol{D}) \boldsymbol{e}_{i} \otimes \boldsymbol{e}_{i}
$$

where $\left\{\mu_{i}\right\}(i=1,2,3)$ are the eigenvalues of $\boldsymbol{F}$.

Define three scalar-valued functions $\phi_{i}(\mathrm{OP}, \boldsymbol{D})(i=0,1,2)$ by

$$
\left(\begin{array}{lll}
1 & \lambda_{1} & \lambda_{1}^{2} \\
1 & \lambda_{2} & \lambda_{2}^{2} \\
1 & \lambda_{3} & \lambda_{3}^{2}
\end{array}\right)\left(\begin{array}{l}
\phi_{0} \\
\phi_{1} \\
\phi_{2}
\end{array}\right)=\left(\begin{array}{l}
\mu_{1} \\
\mu_{2} \\
\mu_{3}
\end{array}\right)
$$

i.e.

$$
\mu_{i}=\sum_{k=0}^{2} \phi_{k} \lambda_{i}^{k}
$$

Such $\phi_{i}(\mathrm{OP}, \boldsymbol{D})(i=0,1,2)$ are well defined since

$$
\operatorname{det}\left|\begin{array}{lll}
1 & \lambda_{1} & \lambda_{1}^{2} \\
1 & \lambda_{2} & \lambda_{2}^{2} \\
1 & \lambda_{3} & \lambda_{3}^{2}
\end{array}\right|=\left(\lambda_{1}-\lambda_{2}\right)\left(\lambda_{2}-\lambda_{3}\right)\left(\lambda_{3}-\lambda_{1}\right) \neq 0
$$

for the distinct $\lambda_{i}$.

After applying (19) and (30), (28) yields

$$
\boldsymbol{F}(\mathrm{OP}, \boldsymbol{D})=\sum_{i=1}^{3} \sum_{k=0}^{2} \phi_{k} \lambda_{i}^{k} \boldsymbol{e}_{i} \otimes \boldsymbol{e}_{i}=\phi_{0} \mathbf{1}+\phi_{1} \boldsymbol{D}+\phi_{2} \boldsymbol{D}^{2} .
$$

By applying (31) for both $\boldsymbol{F}(\mathrm{OP}, \boldsymbol{D})$ and $\boldsymbol{F}\left(\mathrm{OP}, \boldsymbol{Q D} \boldsymbol{Q}^{T}\right)$, (18) yields

$$
\boldsymbol{Q}\left\{\sum_{i=0}^{2}\left[\phi_{i}\left(\mathrm{OP}, \mathbf{Q D} \boldsymbol{Q}^{T}\right)-\phi_{i}(\mathrm{OP}, \boldsymbol{D})\right] \boldsymbol{D}^{i}\right\} \boldsymbol{Q}^{T}=\mathbf{0} \quad \forall \boldsymbol{Q} .
$$

Pre-multiplying (32) by $\mathbf{Q}^{T}$ and post-multiplying by $\mathbf{Q}$ yields

$$
\sum_{i=0}^{2}\left[\phi_{i}\left(\mathrm{OP}, \boldsymbol{Q} \boldsymbol{D} \boldsymbol{Q}^{T}\right)-\phi_{i}(\mathrm{OP}, \boldsymbol{D})\right] \boldsymbol{D}^{i}=\mathbf{0} .
$$

Theorem 6. 1, $\boldsymbol{D}$ and $\boldsymbol{D}^{2}$ are linearly independent if three eigenvalues of $\boldsymbol{D}$ are distinct.

Proof. Suppose that 1, $\boldsymbol{D}$ and $\boldsymbol{D}^{2}$ are linearly dependent, i.e.

$$
\alpha \mathbf{1}+\beta \boldsymbol{D}+\gamma \boldsymbol{D}^{2}=\mathbf{0}
$$

where at least one of $\alpha, \beta$ and $\gamma$ is not zero. This, with aid of (19), yields

$$
\sum_{i=1}^{3}\left(\alpha+\beta \lambda_{i}+\gamma \lambda_{i}^{2}\right) \boldsymbol{e}_{i} \otimes \boldsymbol{e}_{i}=\mathbf{0} .
$$


Then

$$
\sum_{k=1}^{3}\left(\alpha+\beta \lambda_{k}+\gamma \lambda_{k}^{2}\right) \boldsymbol{e}_{k}=\mathbf{0}
$$

in which the mutual orthogonality of $\left\{\boldsymbol{e}_{i}\right\}(i=1,2,3)$ has been used. This leads to

$$
\alpha+\beta \lambda_{k}+\gamma \lambda_{k}^{2}=0 \quad(k=1,2,3)
$$

since $\left\{\boldsymbol{e}_{k}\right\}(k=1,2,3)$ are linearly independent.

Equation (37) shows that each $\lambda_{k}$ is a root of the same quadratic equation. It is obvious that at least two of the $\lambda_{k}$ must be equal. This is contrary to the hypothesis of distinct $\lambda_{k}$. Thus, to satisfy (34), $\alpha, \beta$ and $\gamma$ must all be zero which implies that $\mathbf{1}$, $\boldsymbol{D}$ and $\boldsymbol{D}^{2}$ are linearly independent.

By applying Theorem 6, (33) gives

$$
\phi_{i}(\mathrm{OP}, \boldsymbol{D})=\phi_{i}\left(\mathrm{OP}, \boldsymbol{Q D} \boldsymbol{Q}^{T}\right) \quad(i=0,1,2) \quad \forall \boldsymbol{Q} .
$$

THeOrem 7. The $\phi_{i}$, which satisfy (38), depend on $\boldsymbol{D}$ only through its invariants

$$
\begin{gathered}
I_{1}(\boldsymbol{D})=\operatorname{tr} \boldsymbol{D}=\lambda_{1}+\lambda_{2}+\lambda_{3}, \\
I_{2}(\boldsymbol{D})=\frac{1}{2}\left[\operatorname{tr}^{2} \boldsymbol{D}-\operatorname{tr} \boldsymbol{D}^{2}\right]=\lambda_{1} \lambda_{2}+\lambda_{1} \lambda_{3}+\lambda_{2} \lambda_{3}, \\
I_{3}(\boldsymbol{D})=\operatorname{det} \boldsymbol{D}=\lambda_{1} \lambda_{2} \lambda_{3}
\end{gathered}
$$

where

$$
\operatorname{tr} \boldsymbol{D}^{2}=\operatorname{tr}(\boldsymbol{D} \boldsymbol{D})
$$

Proof. To prove this theorem, we need to prove

$$
\phi_{i}(\mathrm{OP}, \boldsymbol{A})=\phi_{i}(\mathrm{OP}, \boldsymbol{B}) \quad(i=0,1,2)
$$

whenever $I_{k}(\boldsymbol{A})=I_{k}(\boldsymbol{B})(k=1,2,3)$. Here $\boldsymbol{A}$ and $\boldsymbol{B}$ are two symmetric tensors. Suppose $I_{k}(\boldsymbol{A})=I_{k}(\boldsymbol{B})(k=1,2,3)$, then $\boldsymbol{A}$ and $\boldsymbol{B}$ have the same eigenvalues which are donated by $\lambda_{i}(i=1,2,3)$. $\boldsymbol{A}$ and $\boldsymbol{B}$ may thus be represented by

$$
\boldsymbol{A}=\sum_{i=1}^{3} \lambda_{i} \boldsymbol{a}_{i} \otimes \boldsymbol{a}_{i}, \quad \boldsymbol{B}=\sum_{i=1}^{3} \lambda_{i} \boldsymbol{b}_{i} \otimes \boldsymbol{b}_{i}
$$

where $\left\{\boldsymbol{a}_{i}\right\}$ and $\left\{\boldsymbol{b}_{i}\right\}(i=1,2,3)$ are the eigenvectors of $\boldsymbol{A}$ and $\boldsymbol{B}$ respectively.

Define

$$
\boldsymbol{Q}=\boldsymbol{b}_{i} \otimes \boldsymbol{a}_{i}
$$

Then $\boldsymbol{Q}$ is a rotation because

$$
\begin{gathered}
\mathbf{Q} \mathbf{Q}^{T}=\boldsymbol{Q}\left(\boldsymbol{a}_{i} \otimes \boldsymbol{b}_{i}\right)=\boldsymbol{Q} \boldsymbol{a}_{i} \otimes \boldsymbol{b}_{i}=\boldsymbol{b}_{i} \otimes \boldsymbol{b}_{i}=\mathbf{1}, \\
\operatorname{det} \boldsymbol{Q}=\frac{\boldsymbol{Q} \boldsymbol{a}_{1} \cdot \boldsymbol{Q} \boldsymbol{a}_{2} \times \mathbf{Q} \boldsymbol{a}_{3}}{\boldsymbol{a}_{1} \cdot \boldsymbol{a}_{2} \times \boldsymbol{a}_{3}}=1 .
\end{gathered}
$$

Thus

$$
\mathbf{Q} \boldsymbol{A} \boldsymbol{Q}^{T}=\sum_{i=1}^{3} \lambda_{i}\left(\boldsymbol{Q} \boldsymbol{a}_{i} \otimes \boldsymbol{Q} \boldsymbol{a}_{i}\right)=\sum_{i=1}^{3} \lambda_{i}\left(\boldsymbol{b}_{i} \otimes \boldsymbol{b}_{i}\right)=\boldsymbol{B} .
$$


By the hypothesis, (38) holds for all rotations. It must be true for the $\boldsymbol{Q}$ defined by (42). Applying (38) and (43) leads to

$$
\phi_{i}(\mathrm{OP}, \boldsymbol{A})=\phi_{i}(\mathrm{OP}, \boldsymbol{B}) \text {. }
$$

Thus we have

$$
\phi_{i}(\mathrm{OP}, \boldsymbol{D})=\psi_{i}\left(\mathrm{OP}, I_{1}(\boldsymbol{D}), I_{2}(\boldsymbol{D}), I_{3}(\boldsymbol{D})\right) \quad(i=0,1,2) .
$$

Conversely, if (44) holds, then

$$
\phi_{i}\left(\mathrm{OP}, \mathbf{Q D} \mathbf{Q}^{T}\right)=\psi_{i}\left(\mathrm{OP}, I_{1}\left(\mathbf{Q D} \mathbf{Q}^{T}\right), I_{2}\left(\mathbf{Q D} \mathbf{Q}^{T}\right), I_{3}\left(\mathbf{Q D} \mathbf{Q}^{T}\right)\right) \quad(i=0,1,2) .
$$

But

$$
\begin{gathered}
I_{1}\left(\mathbf{Q D} \boldsymbol{Q}^{T}\right)=\operatorname{tr}\left(\mathbf{Q} \boldsymbol{D} \boldsymbol{Q}^{T}\right)=\operatorname{tr}\left(\mathbf{D} \mathbf{Q} \mathbf{Q}^{T}\right)=\operatorname{tr} \boldsymbol{D}=I_{1}(\boldsymbol{D}), \\
I_{2}\left(\mathbf{Q} \boldsymbol{D} \boldsymbol{Q}^{T}\right)=\frac{1}{2}\left[\operatorname{tr}^{2}\left(\mathbf{Q} \boldsymbol{D} \boldsymbol{Q}^{T}\right)-\operatorname{tr}\left(\mathbf{Q} \boldsymbol{D} \boldsymbol{Q}^{T}\right)^{2}\right]=\frac{1}{2}\left[\operatorname{tr}^{2} \boldsymbol{D}-\operatorname{tr}\left(\mathbf{Q} \boldsymbol{D}^{2} \mathbf{Q}^{T}\right)\right] \\
=\frac{1}{2}\left[\operatorname{tr}^{2} \boldsymbol{D}-\operatorname{tr}\left(\boldsymbol{D}^{2} \mathbf{Q} \mathbf{Q}^{T}\right)\right]=\frac{1}{2}\left[\operatorname{tr}^{2} \boldsymbol{D}-\operatorname{tr} \boldsymbol{D}^{2}\right]=I_{2}(\boldsymbol{D}), \\
I_{3}\left(\mathbf{Q} \boldsymbol{D} \boldsymbol{Q}^{T}\right)=\operatorname{det}\left(\mathbf{Q} \boldsymbol{D} \boldsymbol{Q}^{T}\right)=\operatorname{det}^{2} \boldsymbol{Q} \operatorname{det} \boldsymbol{D}=\operatorname{det} \boldsymbol{D}=I_{3}(\boldsymbol{D}) .
\end{gathered}
$$

Thus (44) and (45) lead to (38). Therefore, (44) is both a necessary and sufficient condition for (38).

By applying Theorem 7, (31) can be written as

$$
\boldsymbol{R}=\boldsymbol{F}(\mathrm{OP}, \boldsymbol{D})=\sum_{i=0}^{2} \psi_{i} \boldsymbol{D}^{i}
$$

where

$$
\psi_{i}=\psi_{i}\left(\mathrm{OP}, I_{1}(\boldsymbol{D}), I_{2}(\boldsymbol{D}), I_{3}(\boldsymbol{D})\right)
$$

This is the necessary condition for both the frame indifference of $\boldsymbol{R}$ and the PMFI. It is interesting to note that symmetry of $\boldsymbol{D}$ ensures the symmetry of $\boldsymbol{R}$. This implies that the frame indifference of $\boldsymbol{R}$ and the PMFI together require the symmetry of $\boldsymbol{R}$.

If the three eigenvalues of $\boldsymbol{D}$ are not distinct, we can still obtain (49) by the same method, with $\psi_{1}=\psi_{2}=0$ (for the case of three identical eigenvalues) or $\psi_{2}=0$ (for the case of two identical eigenvalues). Therefore, (49) is valid for all cases.

\subsection{Sufficiency of (49) for both frame indifference of $\boldsymbol{R}$ and PMFI}

Suppose (49) holds, then

$$
\begin{aligned}
& \boldsymbol{F}\left(\mathrm{OP}, \boldsymbol{Q D} \boldsymbol{Q}^{T}\right)=\psi_{0}\left(\mathrm{OP}, I_{1}\left(\mathbf{Q D} \boldsymbol{Q}^{T}\right), I_{2}\left(\mathbf{Q} \boldsymbol{D} \boldsymbol{Q}^{T}\right), I_{3}\left(\mathbf{Q D} \boldsymbol{Q}^{T}\right)\right) \mathbf{1} \\
& +\psi_{1}\left(\mathrm{OP}, I_{1}\left(\mathbf{Q} \boldsymbol{D} \mathbf{Q}^{T}\right), I_{2}\left(\mathbf{Q D} \mathbf{Q}^{T}\right), I_{3}\left(\mathbf{Q D} \boldsymbol{Q}^{T}\right)\right) \mathbf{Q} \boldsymbol{D} \mathbf{Q}^{T} \\
& +\psi_{2}\left(\mathrm{OP}, I_{1}\left(\mathbf{Q} \boldsymbol{D} \mathbf{Q}^{T}\right), I_{2}\left(\mathbf{Q} \boldsymbol{D} \mathbf{Q}^{T}\right), I_{3}\left(\mathbf{Q} \boldsymbol{D} \mathbf{Q}^{T}\right)\right) \mathbf{Q} \boldsymbol{D} \mathbf{Q}^{T} \mathbf{Q} \boldsymbol{D} \mathbf{Q}^{T} \\
& =\psi_{0}\left(\mathrm{OP}, I_{1}(\boldsymbol{D}), I_{2}(\boldsymbol{D}), I_{3}(\boldsymbol{D})\right) \mathbf{Q} \mathbf{1} \mathbf{Q}^{T} \\
& +\psi_{1}\left(\mathrm{OP}, I_{1}(\boldsymbol{D}), I_{2}(\boldsymbol{D}), I_{3}(\boldsymbol{D})\right) \boldsymbol{Q} \boldsymbol{D} \boldsymbol{Q}^{T} \\
& +\psi_{2}\left(\mathrm{OP}, I_{1}(\boldsymbol{D}), I_{2}(\boldsymbol{D}), I_{3}(\boldsymbol{D})\right) \mathbf{Q} \boldsymbol{D}^{2} \mathbf{Q}^{T} \\
& =\mathbf{Q F}(\mathrm{OP}, \boldsymbol{D}) \boldsymbol{Q}^{T}
\end{aligned}
$$

after applying (46)-(48). This establishes the sufficiency of (49) for both the frame indifference of $\boldsymbol{R}$ and the PMFI. Since (49) is both a necessary and sufficient condition, we conclude that (i) every model which does not belong to (49) is not properly invariant; and (ii) every model satisfying (49) is properly invariant. 


\section{Positive semi-definiteness of $\boldsymbol{R}$}

Let $\lambda_{i}$ and $f_{i}(i=1,2,3)$ be the three eigenvalues of the $\boldsymbol{D}$ and $\boldsymbol{R}$ respectively. Since $\boldsymbol{R}$ is related to $\boldsymbol{D}$ by (49), then

$$
f_{i}=\psi_{0}+\psi_{1} \lambda_{i}+\psi_{2} \lambda_{i}^{2} \quad(i=1,2,3) .
$$

Because $\boldsymbol{D}$ and $\boldsymbol{R}$ are real-valued symmetric tensors, $\lambda_{i}$ and $f_{i}(i=1,2,3)$ must be real-valued, that is

$$
\begin{aligned}
& \bar{f}_{i}=f_{i}, \\
& \bar{\lambda}_{i}=\lambda_{i},
\end{aligned}
$$

where the overbar stands for the conjugate. By (51)

$$
\bar{f}_{i}=\bar{\psi}_{0}+\bar{\psi}_{1} \bar{\lambda}_{i}+\bar{\psi}_{2} \bar{\lambda}_{i}^{2}
$$

Substituting (52) and (53) into (54) yields

$$
f_{i}=\bar{\psi}_{0}+\bar{\psi}_{1} \lambda_{i}+\bar{\psi}_{2} \lambda_{i}^{2} .
$$

This, with (51), yields

$$
\left(\psi_{0}-\bar{\psi}_{0}\right)+\left(\psi_{1}-\bar{\psi}_{1}\right) \lambda_{i}+\left(\psi_{2}-\bar{\psi}_{2}\right) \lambda_{i}^{2}=0 \quad \forall \lambda_{i} \in R
$$

which indicates that

$$
\psi_{i}=\bar{\psi}_{i} \quad(i=0,1,2) .
$$

Therefore, $\psi_{i}(i=0,1,2)$ must be real-valued.

The necessary and sufficient condition for a symmetric tensor to be positive semidefinite is that all of its eigenvalues are positive semi-definite. Thus both a necessary and sufficient condition for the realizability is

$$
\psi_{0}+\psi_{1} \lambda_{i}+\psi_{2} \lambda_{i}^{2} \geqslant 0 \quad \forall \lambda_{i} \in R
$$

in which the equality is for the reversible processes in the sense of the second law of thermodynamics while the inequality is for the irreversible processes (Wang 1997). Note that the flow processes are irreversible. We restrict the following analysis for finding the necessary and sufficient conditions for (58) to the case of strict inequality, i.e.

$$
\psi_{0}+\psi_{1} \lambda_{i}+\psi_{2} \lambda_{i}^{2}>0 \quad \forall \lambda_{i} \in R .
$$

Two necessary conditions for (59) can be easily obtained by considering the cases of $\lambda_{i}=0$ and $\left|\lambda_{i}\right| \rightarrow \infty$ respectively as

$$
\begin{aligned}
& \psi_{0}>0, \\
& \psi_{2}>0 .
\end{aligned}
$$

By dividing (59) by $\psi_{0}$, we can rearrange it into the alternative form

$$
\left(1+\frac{\psi_{1} \lambda_{i}}{2 \psi_{0}}\right)^{2}+\left(\frac{\psi_{2}}{\psi_{0}}-\frac{\psi_{1}^{2}}{4 \psi_{0}^{2}}\right) \lambda_{i}^{2}>0 \quad \forall \lambda_{i} \in R .
$$

This yields another necessary condition by setting $\lambda_{i}=-2 \psi_{0} / \psi_{1}$ :

$$
\psi_{1}^{2}-4 \psi_{0} \psi_{2}<0
$$

or

$$
\left|\psi_{1}\right|<2\left(\psi_{0} \psi_{2}\right)^{1 / 2}
$$


Conversely, it is easy to show that (60), (61) and (63) also constitute sufficient conditions for (59). Consequently, we can conclude that (i) every model violating (60), (61) or (63) is incapable of preserving the positive definiteness of the Reynolds stresses, and (ii) every model satisfying (60), (61) and (63) preserves the positive definiteness of the Reynolds stresses. It is worth noting that these conditions must be satisfied not only by every turbulence model, but also in each iteration step of a numerical scheme.

\section{Discussion}

Equations (49), (60), (61) and (63) form a closure model which is both a necessary and sufficient condition for preserving both the invariance and realizability of $\boldsymbol{R}$ and satisfying the PMFI. It differs from those in previous works mainly in following aspects: (i) the previous models (e.g. Speziale 1987; Durbin \& Speziale 1994; Speziale et al. 1994; Pope 1983; Lumley 1978) are only the sufficient condition for the invariance, realizability and/or the PMFI; (ii) it is derived in a mathematically rigorous manner without the introduction of intuitive assumptions in the derivation; (iii) the frame indifference of $\boldsymbol{R}$, the PMFI and the principle of observer transformation are clearly distinguished in its derivation; and (iv) the independent variables are chosen in a proper way.

Several important features are revealed regarding the Reynolds stress closure. First, a properly invariant model cannot depend on the mean velocity itself (Theorem 1). The models permitting such dependence (Lumley 1970; Speziale 1979, 1981, 1982, 1987 and 1991) are thus improperly invariant. It is found, from the present work (equation (49)), that the frame-indifference of $\boldsymbol{R}$ and the PMFI together ensure the symmetry of $\boldsymbol{R}$. The three eigenvectors of $\boldsymbol{R}$ are the same as those of $\boldsymbol{D}$ (Theorem 5). The three corresponding eigenvalues are related to those of $\boldsymbol{D}$ through (51).

The $I_{1}, I_{2}$, and $I_{3}$ formed by $\boldsymbol{D}$ are found to be three characteristic scalars for a properly invariant model. The application of $k, l$ or $\varepsilon$ as the characteristic scalars in the literature comes from intuitive arguments, and needs to be re-examined. Note also that the $I_{1}, I_{2}$, and $I_{3}$ are only functions of $\boldsymbol{D}$. The models using them as the characteristic scalars will be explicit. This would reduce the computational effort.

The present model shows that a properly invariant model cannot involve $\boldsymbol{W}$ explicitly. This confirms the finding by Speziale (1980). However, the models proposed recently by Speziale et al. (1991), Gatski \& Speziale (1993), Ristorcelli et al. (1995), and Xu \& Speziale (1996) depend explicitly on $\boldsymbol{W}$. Though such models were derived based on the consideration of the invariance and the PMFI, they are not properly invariant. This is believed to result from the use of $\boldsymbol{D}$ and $\boldsymbol{W}$ as two independent variables.

For a model to be realizable, it must satisfy (60), (61) and (63). With vanishing $\psi_{2}$ for the $k-l$ or $k-\varepsilon$ models (Speziale 1987), (61) and (63) are violated. Thus the $k-\varepsilon$ and $k-l$ models cannot preserve the realizability. It is well known that the $k-l$ and $k-\varepsilon$ models can give rise to highly inaccurate predictions for the normal Reynolds stresses which make it impossible to describe or predict turbulent channel flow, secondary flow in non-circular ducts and separated turbulent flows (Speziale 1987) in which the normal Reynolds stresses play an important role. Note that the realizability puts a restriction on the models by taking account of normal Reynolds stress properties. The violation of both the necessary and sufficient condition for the realizability may be the reason why they can yield inaccurate predictions for the normal Reynolds stresses. 
As an example, we analyse how well Prandtl's mixing length model preserves the physics. In a tensorial form, it can be written as (Hinze 1959; Schlichting 1968)

$$
\boldsymbol{R}=-p \mathbf{1}+2 \eta_{1} \rho l^{2}\left(2 \operatorname{tr} \boldsymbol{D}^{2}\right)^{1 / 2} \boldsymbol{D}=-p \mathbf{1}+2 \eta_{1} \rho l^{2}\left(2\left(I_{1}^{2}-2 I_{2}\right)\right)^{1 / 2} \boldsymbol{D}
$$

in which $-p=\frac{1}{3} \operatorname{tr} \boldsymbol{R}, \eta_{1}$ is a dimensionless constant, $\rho$ is the density of the fluid, and $l$ is the distance from the wall for wall turbulence. It can be classified as a special case of (49) with $\psi_{0}=-p, \psi_{1}=2 \eta_{1} \rho l^{2}\left(2\left(I_{1}^{2}-2 I_{2}\right)\right)^{1 / 2}$, and $\psi_{2}=0$. Therefore Prandtl's mixing length model is capable of preserving the frame indifference of $\boldsymbol{R}$ and satisfies the PMFI. However, it is incapable of preserving the positive definiteness of $\boldsymbol{R}$ since this violates (61) and (63).

\section{Linear and quadratic theories}

Equation (49), with (60), (61) and (63), forms a general Reynolds stress closure model which is both a necessary and sufficient condition for invariance, realizability and PMFI. Although these equations can be used to judge whether a particular model is properly invariant and/or realizable, they are not convenient to use in finding new specific models because they contain three functions $\psi_{i}(i=0,1,2)$ of $I_{k}(\boldsymbol{D})(k=1,2,3)$. Such functions must be determined through experiments. $\dagger$ However, it is very complicated and difficult (if not impossible) to carry out such experiments because of the varieties of possible motions. That being so, it is natural to introduce some simplifications or approximations to reduce the $\psi_{i}$ to some parameters independent of $I_{k}$ through factoring out the effect of $I_{k}$. The linear and quadratic theories to be developed here are two such approximate theories. Unlike the exact theory, we cannot expect the approximate theories to be valid for any turbulent flows which can be described by (1). However, such approximate theories deserve to be studied because we cannot understand the exact theory without first having understood the approximate theories to which it gives rise. Note that the linear and quadratic relations are usually used to approximate the general nonlinear relation, and are often accurate enough for some turbulent flows.

\subsection{Linear and quadratic theories}

Suppose that we can approximate the relation between $\boldsymbol{R}$ and $\boldsymbol{D}$ by a linear or quadratic form for some turbulent flows in the group described by (1). To obtain an approximate theory, set

$$
\boldsymbol{D}=\epsilon \hat{\boldsymbol{D}}
$$

where

$$
|\hat{\boldsymbol{D}}|=\left(D_{i j} D_{i j}\right)^{1 / 2}=o(1)
$$

and

$$
\epsilon \ll 1 \text {. }
$$

Assuming that $\psi_{i}(i=0,1,2)$ are smooth in $I_{k}(k=1,2,3)$ (physically, this assumes that $\boldsymbol{D}$ affects $\boldsymbol{R}$ in a continuous way; it is a reasonable assumption for practical

$\dagger$ It is believed that all details regarding constitutive relations can be obtained by imposing enough fundamental principles or laws. At the present time, however, the author is unaware of any other fundamental laws which can be used to further constrain $\psi_{i}$ (although we may invoke the Buckingham- $\Pi$ theorem (see Brand 1957) to further limit the form of any expression for $\psi_{i}$.). Therefore, it is only from experiments that they can be determined at the present stage of development of theory. 
turbulent flows), then Taylor expansion of $\psi_{i}$ around $\boldsymbol{D}=\mathbf{0}\left(I_{k}=0 ; k=1,2,3\right)$, gives that

$$
\begin{array}{r}
\psi_{i}\left(\mathrm{OP}, I_{k}\right)=\psi_{i}(\mathrm{OP}, 0)+\left.\sum_{k=1}^{3} I_{k} \frac{\partial \psi_{i}}{\partial I_{k}}\right|_{I_{k}=0}+\left.\sum_{k=1}^{3} \sum_{j=1}^{3} I_{k} I_{j} \frac{\partial^{2} \psi_{i}}{\partial I_{k} \partial I_{j}}\right|_{I_{k}=0}+o\left(I_{k}^{3}\right) \\
(i=0,1,2)
\end{array}
$$

in which $\psi_{i}\left(\mathrm{OP}, I_{k}\right)=\psi_{i}\left(\mathrm{OP}, I_{1}, I_{2}, I_{3}\right) ; \psi_{i}(\mathrm{OP}, 0)=\psi_{i}(\mathrm{OP}, 0,0,0)$, and $I_{k}=0$ means that $I_{1}=I_{2}=I_{3}=0$. Note that

$$
\left.\begin{array}{l}
I_{1}=\operatorname{tr} \boldsymbol{D}=\operatorname{tr}(\epsilon \hat{\boldsymbol{D}})=\epsilon \operatorname{tr} \hat{\boldsymbol{D}}=o(\epsilon), \\
2 I_{2}=\epsilon^{2}\left[(\operatorname{tr} \hat{\boldsymbol{D}})^{2}-\operatorname{tr} \hat{\boldsymbol{D}}^{2}\right]=o\left(\epsilon^{2}\right), \\
I_{3}=\operatorname{det}(\epsilon \hat{\boldsymbol{D}})=\epsilon^{3} \operatorname{det} \hat{\boldsymbol{D}}=o\left(\epsilon^{3}\right) .
\end{array}\right\}
$$

Then

$$
\psi_{i}\left(\mathrm{OP}, I_{k}\right)=\psi_{i}(\mathrm{OP}, 0)+\left.\epsilon \operatorname{tr} \hat{\boldsymbol{D}} \frac{\partial \psi_{i}}{\partial I_{1}}\right|_{I_{k}=0}+\left.\epsilon^{2} I_{2}(\hat{\boldsymbol{D}}) \frac{\partial \psi_{i}}{\partial I_{2}}\right|_{I_{k}=0}+\left.\epsilon^{2} \operatorname{tr} \hat{\boldsymbol{D}}^{2} \frac{\partial^{2} \psi_{i}}{\partial I_{1}^{2}}\right|_{I_{k}=0}+o\left(\epsilon^{3}\right)
$$

and

$$
\begin{gathered}
\psi_{0}\left(\mathrm{OP}, I_{k}\right) \mathbf{1}=\left[\psi_{0}(\mathrm{OP}, 0)+\left.\epsilon \operatorname{tr} \hat{\boldsymbol{D}} \frac{\partial \psi_{0}}{\partial I_{1}}\right|_{I_{k}=0}+\left.\epsilon^{2} I_{2}(\hat{\boldsymbol{D}}) \frac{\partial \psi_{0}}{\partial I_{2}}\right|_{I_{k}=0}+\left.\epsilon^{2} \operatorname{tr} \hat{\boldsymbol{D}}^{2} \frac{\partial^{2} \psi_{0}}{\partial I_{1}^{2}}\right|_{I_{k}=0}\right] \\
\psi_{1}\left(\mathrm{OP}, I_{k}\right) \boldsymbol{D}=\psi_{1}(\mathrm{OP}, 0) \epsilon \hat{\boldsymbol{D}}+\left.\epsilon \operatorname{tr} \hat{\boldsymbol{D}} \epsilon \hat{\boldsymbol{D}} \frac{\partial \psi_{1}}{\partial I_{1}}\right|_{I_{k}=0}+o\left(\epsilon^{3}\right), \\
\psi_{2}\left(\mathrm{OP}, I_{k}\right) \boldsymbol{D}^{2}=\psi_{2}(\mathrm{OP}, 0) \epsilon^{2} \hat{\boldsymbol{D}}^{2}+o\left(\epsilon^{3}\right) .
\end{gathered}
$$

Retaining terms up to $o(\epsilon)$ and $o\left(\epsilon^{2}\right)$ respectively yields a linear theory for (49)

$$
\boldsymbol{R}_{l}=[a(\mathrm{OP})+b(\mathrm{OP}) \operatorname{tr} \boldsymbol{D}] \mathbf{1}+c(\mathrm{OP}) \boldsymbol{D},
$$

and a quadratic theory

$$
\begin{aligned}
\boldsymbol{R}_{q}=[a(\mathrm{OP})+b(\mathrm{OP}) \operatorname{tr} \boldsymbol{D}+ & \left.d(\mathrm{OP}) I_{2}(\boldsymbol{D})+e(\mathrm{OP}) \operatorname{tr}^{2} \boldsymbol{D}\right] \mathbf{1} \\
& +[c(\mathrm{OP})+f(\mathrm{OP}) \operatorname{tr} \boldsymbol{D}] \boldsymbol{D}+g(\mathrm{OP}) \boldsymbol{D}^{2}
\end{aligned}
$$

where

$$
\left.\begin{array}{ll}
a(\mathrm{OP})=\psi_{0}(\mathrm{OP}, 0), & b(\mathrm{OP})=\left.\frac{\partial \psi_{0}}{\partial I_{1}}\right|_{I_{k}=0}, \\
c(\mathrm{OP})=\psi_{1}(\mathrm{OP}, 0), & d(\mathrm{OP})=\left.\frac{\partial \psi_{0}}{\partial I_{2}}\right|_{I_{k}=0}, \\
e(\mathrm{OP})=\left.\frac{\partial^{2} \psi_{0}}{\partial I_{1}^{2}}\right|_{I_{k}=0}, \quad f(\mathrm{OP})=\left.\frac{\partial \psi_{1}}{\partial I_{1}}\right|_{I_{k}=0}, \\
g(\mathrm{OP})=\psi_{2}(\mathrm{OP}, 0)
\end{array}\right\}
$$

are functions of some scalar-valued parameters OP which are independent of $\boldsymbol{D}$. It is clear that the higher-order theory will introduce more parameters. Such parameters are much easier to determine through experiments because of their independence of $D$. Furthermore, we can invoke other fundamental laws such as the Buckingham- $\Pi$ theorem (Brand 1957) to further simplify the experiments. 
5.2. Positive definiteness

By making use of (60), (61) and (63), we have

$$
\begin{gathered}
a>0, \\
g>0, \\
c^{2}-4 a g<0 .
\end{gathered}
$$

If we approximate $\boldsymbol{R}$ by $\boldsymbol{R}_{l}$ or $\boldsymbol{R}_{q}$, the realizability requires that

$$
f_{i}=a+b \operatorname{tr} \boldsymbol{D}+c \lambda_{i}>0 \quad(i=1,2,3),
$$

or

$$
f_{i}=a+b \operatorname{tr} \boldsymbol{D}+d I_{2}(\boldsymbol{D})+e \operatorname{tr}^{2} \boldsymbol{D}+(c+f \operatorname{tr} \boldsymbol{D}) \lambda_{i}+g \lambda_{i}^{2}>0 \quad(i=1,2,3) .
$$

Since $\boldsymbol{R}_{l}$ and $\boldsymbol{R}_{q}$ are just approximations of (49), we cannot expect (80) and (81) to be valid for all $\lambda_{i}, \operatorname{tr} \boldsymbol{D}, I_{2} \in R$. However, they should be satisfied by all $\lambda_{i}, \operatorname{tr} \boldsymbol{D}$, and $I_{2}$ of a class of turbulent flows for which the Reynolds stresses can be approximated by $\boldsymbol{R}_{l}$ or $\boldsymbol{R}_{q}$.

Although the coefficients $a-g$ in $\boldsymbol{R}_{l}$ and $\boldsymbol{R}_{q}$ are related to $\psi_{i}(i=0,1,2)$ by (76), they are not expected to be determined by $(76)$ as $\psi_{i}(i=0,1,2)$ are much more difficult to determine experimentally. They should be determined directly through experiments, and be regarded as empirical coefficients like those in the previous models such as Prandtl's mixing-length, $k-\varepsilon, k-l$, effective-viscosity, and nonlinear $k-\varepsilon$ and $k-l$ models (Speziale 1987). As a matter of fact, $\boldsymbol{R}_{l}$ and $\boldsymbol{R}_{q}$ are similar to these previous models in the sense that they all relate the Reynolds stresses to $\boldsymbol{D}$ in a linear or quadratic manner through some experimentally determined coefficients. The main differences are however that: (i) the $I_{1}$ and $I_{2}$ (not $k, l$ or $\varepsilon$ ) are used as the characteristic scalars in $\boldsymbol{R}_{l}$ and $\boldsymbol{R}_{q}$, and (ii) $\boldsymbol{R}_{l}$ and $\boldsymbol{R}_{q}$ satisfy both necessary and sufficient conditions for the invariance, realizability and PMFI approximately. Thus $\boldsymbol{R}_{l}$ and $\boldsymbol{R}_{q}$ yield a better prediction than the previous linear and quadratic models in the literature.

After analysing the invariance of second-order turbulence closure models, Speziale addressed an interesting question: "Why not mathematically model the Reynolds stresses directly?' (Speziale 1979). Indeed, we have less a priori knowledge about the higher turbulence correlation introduced by the second-order approach than the Reynolds stresses themselves (Speziale 1979). The results obtained in the present work are believed to be useful in modelling the Reynolds stresses directly because (49), (60), (61) and (63), and the linear and quadratic theories developed in the present work form the conditions which must be satisfied by any specific properly invariant and realizability-preserving model.

\section{Concluding remarks}

For a class of turbulent flows for which the Reynolds stresses can be described by (1), both necessary and sufficient conditions are derived in a systematic, rigorous way for the invariance, realizability and PMFI. This leads to a general model (49) with three real-valued functions $\psi_{i}(i=0,1,2)$ satisfying $(60),(61)$ and (63). The results are also valid for the higher-order turbulence correlations and the SGS Reynolds stresses occurring in large-eddy simulations (LES). Any specific model satisfying (49), (60), (61) and (63) is properly invariant and realizable while a model violating these 
conditions is not. The work is believed to be important both for developing specific, properly invariant and realizable models and for clarifying some confusion in the literature by noting that the previous works employ an intuitive approach with the focus on only obtaining the sufficient condition.

To simplify the experiments for finding the specific, properly invariant and realizable closure models, two approximate theories, a linear theory and a quadratic theory, are developed. In these two approximate theories, the Reynolds stress-strain relations are approximated by a linear relation and by a quadratic relation respectively. Such an approximation can be easily extended to higher order. All such approximate theories reduce the modelling to determining some parameters which are independent of mean velocity strain.

It appears necessary to re-examine the following concepts and methods prevalent in the literature: (i) the invariance of the Navier-Stokes equations and the Reynolds stress transport equations; (ii) the confusion about the frame indifference of the Reynolds stresses and the higher-order correlations, the PMFI, and the principle of observer transformation; (iii) the use of some quantities which are dependent as the independent variables; and (iv) the use of turbulent kinetic energy $k$ and the turbulence dissipation rate $\varepsilon$ (or the length scale of turbulence $l$ ) as the characteristic scalars in relating the Reynolds stresses to the rates of strain and local scalar quantities.

The present work confirms some previous intuitive arguments in the literature such as (i) the independence of $\boldsymbol{R}$ of $\boldsymbol{v}$, (ii) the independence of $\boldsymbol{R}$ of $\boldsymbol{W}$, and (iii) the symmetry of $\boldsymbol{R}$, etc.

The $I_{1}, I_{2}$ and $I_{3}$ defined by (39)-(41) are found to be three characteristic scalars relating the Reynolds stresses to the rates of strain and local scalar quantities. The eigenvectors of $\boldsymbol{R}$ are the same as those of $\boldsymbol{D}$ with the associated eigenvalues related to those of $\boldsymbol{D}$ by (51). Prandtl's mixing length model, the $k-l$ model, the $k-\varepsilon$ model, and some models recently proposed in the literature are examined in terms of their invariance, realizability and satisfaction of the PMFI with respect to both the necessary and sufficient conditions. While they may satisfy one or two of the three requirements, no one can simultaneously satisfy the invariance, the realizability and the PMFI. The violation of both the necessary and sufficient condition for the realizability is believed to be the reason why the $k-l$ and $k-\varepsilon$ models yield inaccurate predictions for the normal Reynolds stress.

The author is indebted to one of the anonymous referees for his critical review and constructive comments/suggestions on the original manuscript.

\section{REFERENCES}

Brand, L. 1957 The Pi theorem of dimensional analysis. Arch. Rat. Mech. Anal. 1, 35-45.

Crofalo, M. 1994 Large-eddy simulation: a critical survey of models and applications. Adv. Heat Transfer 25, 321-419.

Durbin, P. A. \& Speziale, C. G. 1994 Realizability of second-moment closure via stochastic analysis. J. Fluid Mech. 280, 395-407.

Gatski, T. B., Sarkar, S. \& Speziale, C. G. (ed.) 1992 Studies in Turbulence. Springer.

Gatski, T. B. \& Speziale, C. G. 1993 On explicit algebraic stress models for complex turbulent flows. J. Fluid Mech. 254, 59-78.

HinZE, J. O. 1959 Turbulence, p. 277. McGraw-Hill.

Lesieur, M. \& MÉtais, O. 1996 New trends in large-eddy simulations of turbulence. Ann. Rev. Fluid Mech. 28, 45-82.

Lumley, J. L. 1970 Toward a turbulent constitutive relation. J. Fluid Mech. 41, 413-434. 
Lumley, J. L. 1978 Computational modeling of turbulent flows. Adv. Appl. Mech. 18, 123-176.

LumLey, J. L. 1983 Turbulence modeling. Trans. ASME: J. Appl. Mech. 50, 1097-1103.

Lumley, J. L. (ed.) 1990 Whither Turbulence? Turbulence at the Crossroads. Springer.

Mason, P. J. 1994 Large-eddy simulation: a critical review of the technique. Q. J. R. Met. Soc. 120, $1-26$.

Morris, W. D. 1981 Heat Transfer and Fluid Flow in Rotating Coolant Channels. Wiley.

Pope, S. B. 1975 A more general effective-viscosity hypothesis. J. Fluid Mech. 72, 331-340.

Pope, S. B. 1983 Consistent modeling of scalars in turbulent flow. Phys. Fluids 26, 404-408.

Ristorcelli, J. R., Lumley, J. L. \& ABID, R. 1995 A rapid-pressure covariance representation consistent with the Taylor-Proudman theorem materially frame indifferent in the two-dimensional limit. J. Fluid Mech. 292, 111-152.

Schlichting, H. 1968 Boundary-Layer Theory, 6th edn, p. 545. McGraw-Hill.

SchumanN, U. 1977 Realizability of Reynolds-stress turbulence models. Phys. Fluids 20, 721-725.

SchumanN, U. 1995 Stochastic backscatter of turbulence energy and scalar variance by random subgrid-scale fluxes. Proc. R. Soc. Lond. A 451, 293-318.

Smith, G. F. 1971 On isotropic functions of symmetric tensors, skew-symmetric tensors and vectors. Intl J. Engng Sci. 9, 899-916.

Speziale, C. G. 1979 Invariance of turbulent closure models. Phys. Fluids 22, 1033-1037.

SPEZIALE, C. G. 1980 Closure relations for the pressure-strain correlation of turbulence. Phys. Fluids 23, 459-463.

Speziale, C. G. 1981 Some interesting properties of two-dimensional turbulence. Phys. Fluids 24, $1425-1427$.

SPEZIALE, C. G. 1982 On turbulent secondary flows in pipes of noncircular cross-section. Intl J. Engng Sci. 20, 863-872.

Speziale, C. G. 1983 Closure Models for rotating two-dimensional turbulence. Geophys. Astrophys. Fluid Dyn. 23, 69-84.

Speziale, C. G. 1984 Material frame-indifference in turbulence modelling. Trans. ASME: J. Appl. Mech. 51, 942-944.

Speziale, C. G. 1985 Galilean invariance of subgrid-scale stress models in the large-eddy simulation of turbulence. J. Fluid Mech. 156, 55-62.

Speziale, C. G. 1987 On nonlinear $K-l$ and $K-\epsilon$ models of turbulence. J. Fluid Mech. 178, 459-475.

Speziale, C. G. 1989 Turbulence modeling in noninertial frames of reference. Theoret. Comput. Fluid Dyn. 1, 3-19.

Speziale, C. G. 1991 Analytical methods for the development of Reynolds-stress closures in turbulence. Annu. Rev. Fluid Mech. 23, 107-157.

Speziale, C. G., Abid, R. \& Durbin, P. A. 1993 New results on the realizability of Reynolds stress turbulence closures. ICASE Rep. 93-76.

Speziale, C. G., Abid, R. \& Durbin, P. A. 1994 On the realizability of Reynolds stress turbulence closures. J. Sci. Comput. 9, 369-403.

Speziale, C. G., Sarkar, S. \& Gatski, T. B. 1991 Modelling the pressure-strain correlation of turbulence: an invariant dynamical systems approach. J. Fluid Mech. 227, 245-272.

Truesdell, C. 1966 Six Lectures on Modern Natural Philosophy, pp. 6, 97. Springer.

Truesdell, C. 1977 A First Course in Rational Continuum Mechanics, pp. 37-50. Academic.

Vreman, B., Geurts, B. \& Kuerten, H. 1994 Realizability conditions for the turbulent stress tensor in large-eddy simulations. J. Fluid Mech. 278, 351-362.

WANG, L. 1997 Second law of thermodynamics and positive semi-definiteness of Reynolds stresses (to be published).

Xu, X. H. \& Speziale, C. G. 1996 Explicit algebraic stress model of turbulence with anisotropic dissipation. AIAA J. 34, 2186-2189. 